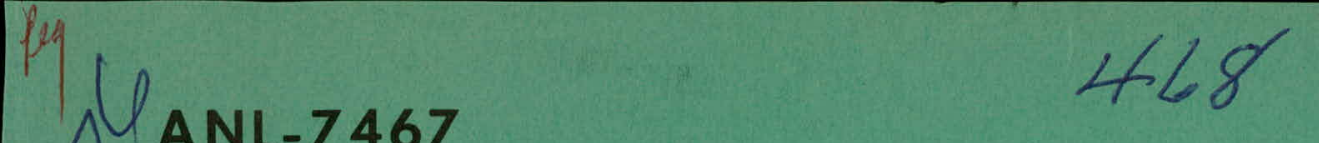

Argonne Mational Laboratorn

INTERFERING RESONANCES

by

P. A. Moldauer 


\section{DISCLAIMER}

This report was prepared as an account of work sponsored by an agency of the United States Government. Neither the United States Government nor any agency Thereof, nor any of their employees, makes any warranty, express or implied, or assumes any legal liability or responsibility for the accuracy, completeness, or usefulness of any information, apparatus, product, or process disclosed, or represents that its use would not infringe privately owned rights. Reference herein to any specific commercial product, process, or service by trade name, trademark, manufacturer, or otherwise does not necessarily constitute or imply its endorsement, recommendation, or favoring by the United States Government or any agency thereof. The views and opinions of authors expressed herein do not necessarily state or reflect those of the United States Government or any agency thereof. 


\section{DISCLAIMER}

Portions of this document may be illegible in electronic image products. Images are produced from the best available original document. 
The facilities of Argonne National Laboratory are owned by the United States Government. Under the terms of a contract (W-31-109-Eng-38) between the U. S. Atomic Energy Commission, Argonne Universities Association and The University of Chicago, the University employs the staff and operates the Laboratory in accordance with policies and programs formulated, approved and reviewed by the Association.

\section{MEMBERS OF ARGONNE UNIVERSITIES ASSOCIATION}

The University of Arizona

Carnegie-Mellon University

Case Western Reserve University

The University of Chicago

University of Cincinnati

Illinois Institute of Technology

University of Illinois

Indiana University

Iowa State University

The University of Iowa
Kansas State University The University of Kansas Loyola University Marquette University Michigan State University The University of Michigan University of Minnesota University of Missouri Northwestern University University of Notre Dame
The Ohio State University Ohio University

The Pennsylvania State University

Purdue University

Saint Louis University

Southern Illinois University

University of Texas

Washington University

Wayne State University

The University of Wisconsin

\section{LEGAL NOTICE}

This report was prepared as an account of Government sponsored work. Neither the United States, nor the Commission, nor any person acting on behalf of the Commission:

A. Makes any warranty or representation, expressed or implied, with respect to the accuracy, completeness, or usefulness of the information contained in this report, or that the use of any information, apparatus, method, or process disclosed in this report may not infringe privately owned rights; or

B. Assumes any liabilities with respect to the use of, or for damages resulting from the use of any information, apparatus, method, or process disclosed in this report.

As used in the above, "person acting on behalf of the Commission" includes any employee or contractor of the Commission, or employee of such contractor, to the extent that such employee or contractor of the Commission, or employee of such contractor prepares, disseminates, or provides access to, any information pursuant to his employment or contract with the Commission, or his employment with such contractor.

Printed in the United States of America

Available from

Clearinghouse for Federal Scientific and Technical Information National Bureau of Standards, U. S. Department of Commerce

Springfield, Virginia 22151

Price: Printed Copy $\$ 3.00 ;$ Microfiche $\$ 0.65$ 
ANL-7467

Physics (TID-4500)

AEC Research and

Development Report

ARGONNE NATIONAL LABORATORY

9700 South Cass Avenue

Argonne, Illinois 60439

INTERFERING RESONANCES

by

P. A. Moldauer

Reactor Physics Division

\section{LE S A L N U I I E}

政 States, nor the Commission

A. Makes any warranty or representation, expressed or implied, with respect to the accuracy, completeness, or usefulness of the information contained in this report, may not infringe racy, completention, apparatus, method, or process disclosed in this report may not infing privately owned rights; or

ately or the B. Assumes any liabiltties with respect to the use of, or fisclosed in this report.

use of any information, apparatus, method, or proceds dif commission" includes any emAs used in the above, "persion acting on behalf of the contractor, to the extent that ployee or contractor of the Commission, or employe or employee of such contractor prepares, such employee or contractor of the Conming disseminates, or provides access to, any with such contractor.

with the Commission, or his

?... - . .

.............

July 1968 


\section{TABLE OF CONTENTS}

$\underline{\text { Page }}$

I. UNRESOLVED RESONANCES . . . . . . . . . . . . . . . 1

II. ISOLATED RESONANCES . . . . . . . . . . . . . . 2

III. INTERFERING RESONANCES. . . . . . . . . . . . . 4

IV. POLE STATISTICS ....................... 8

V. CROSS SECTIONS. . . . . . . . . . . . . 13

VI. AVERAGE CROSS SECTIONS. .................... 15

REFERENCES. . . . . . . . . . . . . 16 


\title{
INTERFERING RESONANCES
}

by

\author{
P. A. Moldauer
}

\section{UNRESOLVED RESONANCES}

Neutron resonances may be unresolved for a variety of reasons. Some resonances are unresolved because of insufficient experimental energy resolution or because of thermal broadening. Some resonances are unresolved because they overlap with resonances in other unseparated isotopes, or they may overlap with resonances belonging to a different angular momentum in the same isotope.

I would like to discuss a quite different cause for unresolved resonances. This is the circumstance in which resonances of the same angular momentum in a single isotope have widths whose magnitudes approach and eventually exceed the spacings of such resonances. It is quite inadequate to describe such a situation in terms of overlapping compound nucleus resonances: The cross section behavior is now dominated by the consequences of interference between resonance terms. Perhaps even more important, and less widely understood, is the fact that, as we shall see, even the values and relations among resonance parameters are dominated by interference effects.

Before discussing the details of such interfering resonances, let me list, for a few elements, the neutron energies above which resonance interference may be said to become overwhelmingly important. This information is in Fig. 1. We see that interference becomes crucial anywhere between about $50 \mathrm{keV}$ in the region of the s-wave strength function peak to $1 \mathrm{MeV}$ for even isotopes in between strength function peaks. Otherwise, energies of two to five hundred $\mathrm{keV}$ are typical. 


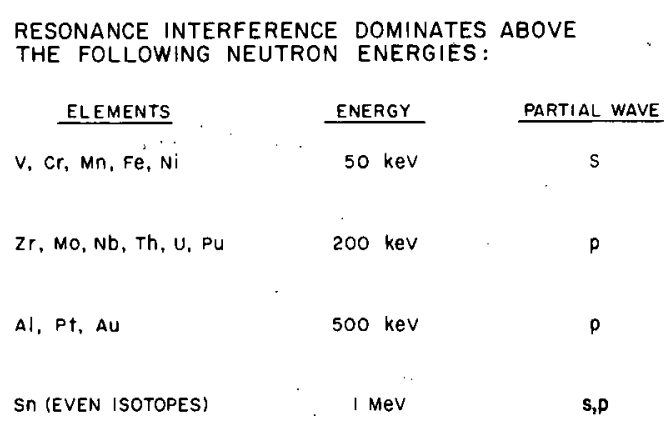

Figure 1

\section{ISOLATED RESONANCES}

In order to deal with the problem of interfering resonances, let us first review the main features of isolated, or non-interfering, resonances. A single isolated neutron scattering resonance is caused. by: a compound state with energy, $E_{O}$, and decay width, $\Gamma$; and these two parameters, together with the potential scattering phase shift, $\phi$, suffice to describe the resonance scattering cross section. How this works is shown in Fig. 2 .

The cross section is written as the absolute square of a scattering amplitude, which we write as one minus the scattering function $\mathrm{S}$. In the many-channel. case, $S$ becomes a scattering matrix. The resonance part of this S-function has the familiar Breit-Wigner form. This means that, considered as an analytic function in the complex energy plane, $S$ has a pole at $E_{0}-\frac{1}{2} i \Gamma$ with a residue of $-i \Gamma e^{-2 i \phi}$. Such a pole is indicated by the dot in the horizontal energy plane in the drawing in this figure.

\section{SINGLE RESONANCE NEUTRON SCATTERING}

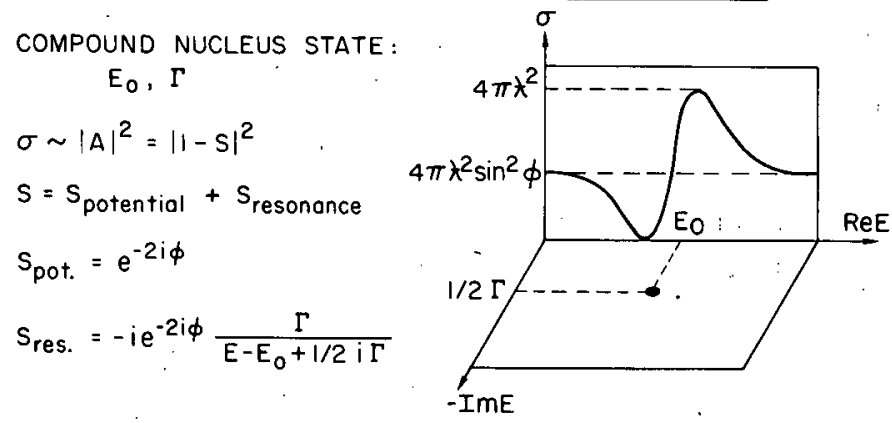

Figure 2 
The resulting expression for the cross section is very familiar but complicated looking. We therefore content ourselves here with drawing a typical cross section curve in the vertical cross section - energy plane. The main thing we need to remember is that associated with any pole of $S$ is a bump in the cross section whose width is related to $\Gamma$ and whose area is related to the magnitude of the residue.

Figure 3 shows what happens when we have many isolated resonances. The $S$ function now has a sum over pole terms, each of which is associated with a cross section resonance bump of characteristic position, width, and area. If such isolated resonances are unresolved because of broadening or overlapping, then we need to know something about the statistical distribution of these resonances and their widths in order to calculate average cross sections, resonance integrals, cross section fluctuations, Doppler effect, etc.

MANY ISOLATED RESONANCES

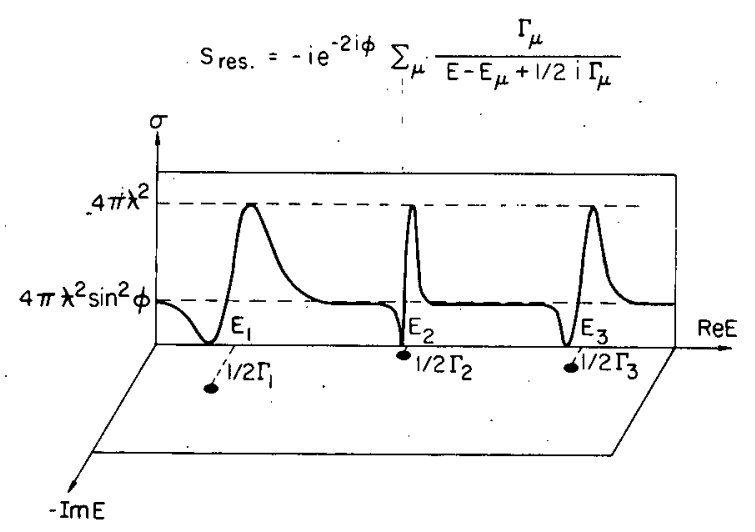

Figure 3

These statistical properties of isolated compound nuclear states have been studied extensively by a large number of people including Wigner, Porter, Thomas, Dyson, Mehta, and others. The results are summarized in Fig. 4.

The mean spacing, $D$, of such non-interfering resonances can be calculated from the spacing of states in a gas of independent fermions. Here $U$ is the total excitation energy of the compound nucleus, and a is a parameter related to the nuclear temperature. Next; the mean width-tospacing ratio is proportional to the optical model transmission 
coefficient, T. The optical mode1, in turn, specifies the energy average of the S-function. The distribution of resonance spacings about their average value is approximated by the Wigner distribution which assigns a zero probability to zero spacings. This is the so-called level repulsion effect. The widths follow the very broad Porter-Thomas distribution according to which the partial widths are distributed over a very large range of values.

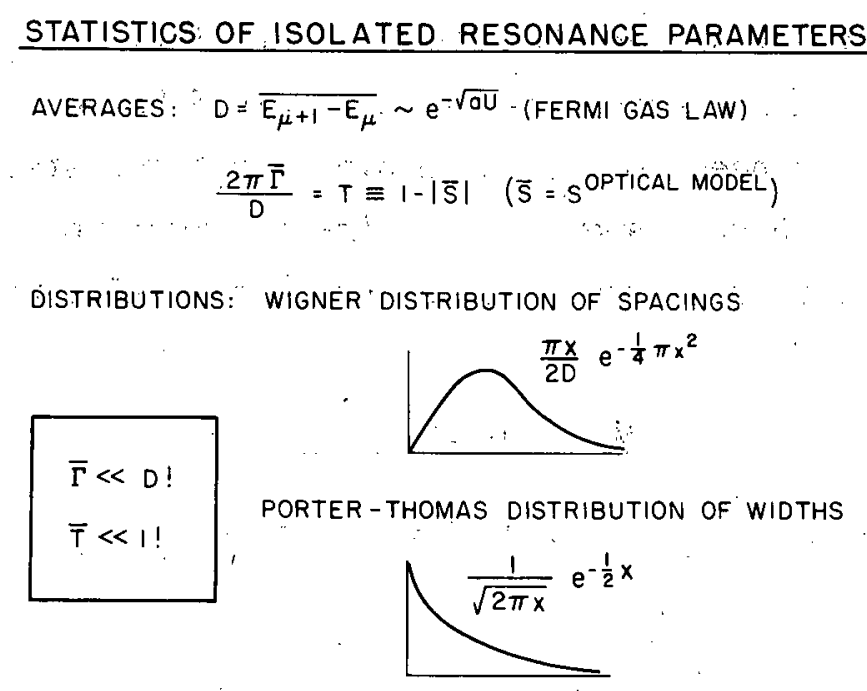

Figure 4

It needs to be stressed, however, that both the derivations and the verifications of all these familiar facts are limited to the case of non-interfering resonances, that is, to cases where $\bar{\Gamma} \ll D$, and hence $\mathrm{T} \ll 1$. When these requirements are fulfilled, we have then a complete statistical description of the resonances, once we know the nuclear temperature and the optical model transmission coefficient.

\section{INTERFERING RESONANCES}

What happens now as the energy increases until the widths become comparable to the spacings? Can we also give a statistical description of interfering resonances? The customary procedure has been to extend the compound nucleus resonance model into the interference region by writing the $\mathrm{S}$-function also there as a sum over compound nucleus resonance poles 
with the same statistical properties as before. However, in the interference region it is really no longer correct to talk about scattering as proceeding through compound nucleus formation. Once $\Gamma$ becomes comparable to $2 \pi \mathrm{D}$, the "compound states" decay before they have had time to become fully formed, and therefore the model becomes self-contradictory.

We are forced, therefore, to approach the problem more formaily'. When we do this, we find that the fluctuating part of the S-function can still be written in the form of a pole expansion as shown at the top of Fig. 5. The big difference between this general expression and the previous one is that now the value of each residue, $\dot{g}_{\mu}{ }^{2}$, is not fixed by the value of the corresponding width, $\Gamma_{\mu}$, but may assume a large range of complex values. We a1so no longer know the relationship of these parameters to the nuclear temperature and transmission coefficient, and we don't know their distributions. These questions are precisely what I would like to discuss now.

$$
\begin{aligned}
& \text { INTERFERING RESONANCES } \\
& S_{\text {res. }}=\Sigma_{\mu} \frac{g_{\mu}^{2}}{E-E_{\mu_{v}}+1 / 2 i \Gamma_{\mu}} \quad \begin{array}{c}
\text { (HUMBLET-ROSENFELD, } \\
\text { KAPUR-PEIERLS) }
\end{array} \\
& \text { DIFFICULTY: } S=e^{-2 i \delta} \quad \text { (UNITARITY) } \\
& \text { SOLUTION: } \quad S=\frac{1-i K}{1+i k} e^{-2 i \phi}, \quad K=\Sigma_{\mu} \frac{F_{\mu}}{E-\varepsilon_{\mu}} \\
& \text { BONUS: }\left(E_{\mu} F_{\mu}\right) \text { DESCRIBE EQUILIBRIUM SYSTEM (R-MATRIX STATES) } \\
& \begin{aligned}
\text { STATISTICAL MECHANICS } \Longrightarrow & \text { FERMI, WIGNER, } \\
& \text { P'ORTER-THOMAS }
\end{aligned}
\end{aligned}
$$

Figure 5

The form of the S-function shown here has been derived by Kapur and Peierls and more recently by Humblet and Rosenfeld. While the meaning of this expression is slightly different in these two formalisms, we can take them to be equivalent here for practical purposes. A cross section fitting procedure which is based on this formula has recently been constructed by Adler and Adler. We may therefore phrase the problem at hand as follows: What is the statistics of resonance pole parameters in the 
Kapur-Peierls or Humblet-Rosenfeld formalisms, or how should one choose parameters for the Adler-Adler program?

In looking at this formula it would appear that we have gained two new free parameters per pole, namely the complex residue, $g_{\mu}{ }^{2}$. However, this is illusory, because $S$ must still have unit magnitude or be a unitary matrix in the many-channel case. This is required by flux conservation. This requirement presents a difficulty, because it is not easily seen exactly what relationships must be satisfied among all the pole parameters, in order that $S$ have unit absolute value, or be unitary, at every value of the energy.

This difficulty can be resolved formally by expressing $S$ in terms of the real reactance, $K$, which has real poles, $E_{\mu}$, and real residues, $F_{\mu}$, and hence the same number of independent pole parameters as we had in the isolated resonance sum. In the many-channel case, $\mathrm{K}$ is a real symmetric matrix. This K-matrix is essentially proportional to Wigner's R-matrix, and they share most of the same properties.

For our purposes, the K- or R-matrix approach yields an additional bonus. By means of R-matrix theory one can relate the K-matrix poles and residues to the energy eigenvalues and eigenfunctions of an artificially defined compound nuclear system having only non-decaying equilibrium states. We can apply the methods of equilibrium statistical mechanics to derive the statistical properties of these eigenvalues and eigenfunctions, and hence of the K-matrix poles and residues.

These properties are precisely the same ones as those of the isolated non-interfering resonance poles: The K-matrix pole density is expected to follow the fermion gas law, the spacing distribution is expected to resemble the Wigner distribution with its level repulsion, and the residues, $F_{\mu}$, have the Porter-Thomas distribution. One can also relate the mean $K$-matrix residue to pole spacing ratio to the optical model transmission coefficient. That expression turns out to be

$$
\mathrm{T}=\frac{\tau}{\left(1+\frac{1}{4} \tau\right)^{2}} \text {, where } \tau=4 \pi \bar{F}_{\mu} / \mathrm{D} \text {. }
$$


If we now construct a K-matrix whose poles and residues have these distributions, we can calculate from it the correct unitary S-matrix and find out what are the distributions and correlations of the S-matrix resonance pole energies, widths, and residues, and how they are related to nuclear temperature and optical model parameters.

The result of one such calculation is shown in Fig. 6. Here we made up a 20 -channel $\mathrm{K}$-matrix whose 50 poles and their residues were chosen, by means of random number generators, to be samples of the appropriate R-matrix statistical distributions. We then calculated the S-matrix whose poles are indicated, as before, as dots in the horizontal energy plane. The bars erected on these dots indicate the magnitudes of the residues in one particular channel, and the cross section curve plotted in the vertical plane is the elastic scattering cross section in that same channel. The triangles in the cross section plane indicate the widths and areas of the bumps that each of the poles would produce if they were isolated resonances. We see that interference produces a pattern of bumps in which it is difficult to recognize the effects of any one pole term. Later we will see, however, that the contribution of the one very strong pole can still be recognized in the energy averaged cross section.

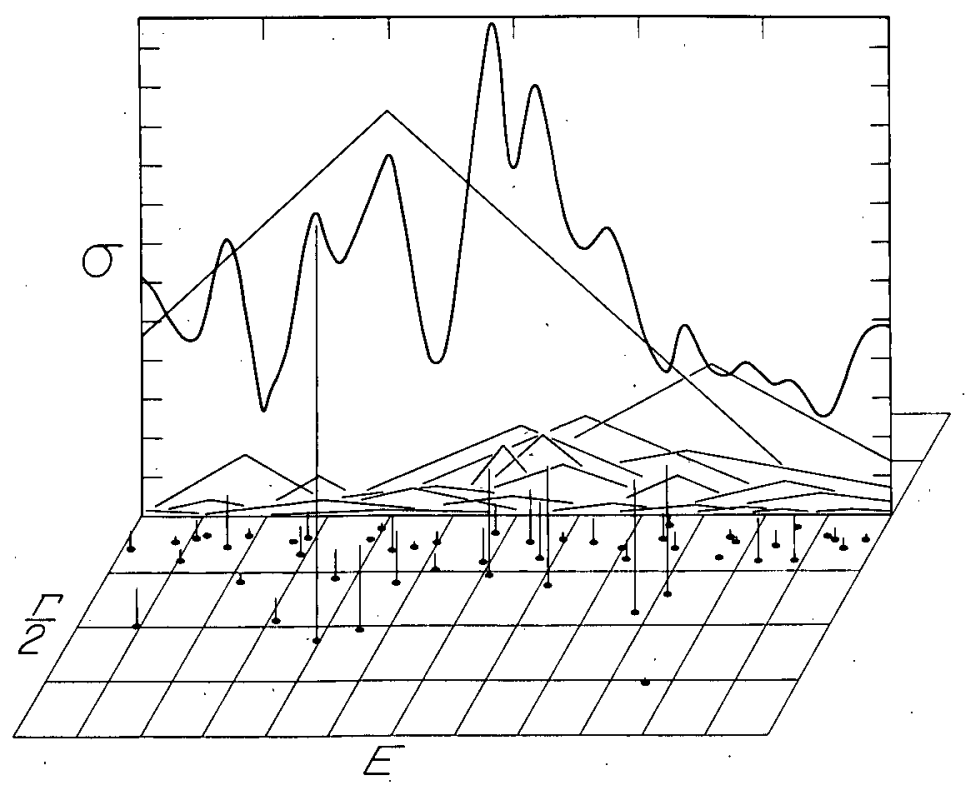

Figure 6 


\section{POLE STATISTICS}

Clearly, we must go beyond the information available from one such statistical pole sample. We want to be able to determine the average resonance pole spacings, widths, and residues as well as their distributions and correlations for arbitrary numbers of open channels with arbitrary optical mode1 transmission coefficients.

We have attacked these problems by three different methods. First of all, analytically; secondly, by means of periodic models of the K-matrix, consisting of sums of appropriate trigonometric functions which permit the calculation of the S-matrix poles in closed form; and, thirdly, we have carried out a number of numerical Monte Carlo calculations of the type just described in the last section. All three methods agree on the averages of the S-matrix resonance pole parameters. These results are summarized in Fig. 7.

\section{STATISTICS OF INTERFERING RESONANCE PARAMETERS}

$$
\begin{aligned}
\text { AVERAGES: } & D>D_{\text {FermI }} \quad \text { (level shift) } \\
& \frac{2 \pi \bar{\Gamma}}{D}=\ln \frac{1}{1-T}, \quad T=1-\mathrm{e}^{-2 \pi \bar{\Gamma} / 0} \\
& \frac{2 \pi\left|\overline{g^{2}}\right|}{D}=\frac{T}{\sqrt{1-T}}, \quad G=\overline{\left|g^{2}\right|}, \quad \frac{2 \pi G}{D} \geq \frac{T}{\sqrt{1-T}}
\end{aligned}
$$

\section{Figure 7}

First of all, because of the cumulative effect of the energy dependent level shift, the mean spacing of S-matrix poles is generally greater than the mean spacing calculated from the fermion gas law, and this difference increases with increasing energy. It must be calculated in each case from the level shifts in all channels.

The average width-to-spacing ratio is proportional to the logarithm of $1 / 1-T$, and the magnitude of the average residue-to-spacing ratio 
is proportional to $\mathrm{T} / \sqrt{1-\mathrm{T}}$. In many-channel cases these two relations are satisfied for each channel separately, that is, for the average par-. tial widths and the average channel residues.

The average of the absolute values of the channel residues is of considerable interest for average cross section calculations. This quantity is, of course, affected by the distribution of the phases of the residues, regarding which we have only numerical information so far. Hence the inequality in the last expression in this figure.

In the limit of small transmission coefficients, a11 of these expressions become identical to the isolated resonance compound nucleus relations which hold for non-interfering resonances and which we discussed earlier.

The two-boxed relations are plotted in Fig. 8. They are the top curves for the average width-to-spacing ratio on the left and for the average residue-to-spacing ratio on the right. The points are the results of numerical calculations with one of the periodic K-matrix models. Each of the bottom curves represents the result of the traditional linear extrapolation of the isolated resonance compound nucleus formulae. I would like to ignore the middle curves here. They result from another theory. Please note, however, the logarithmic vertical scale.

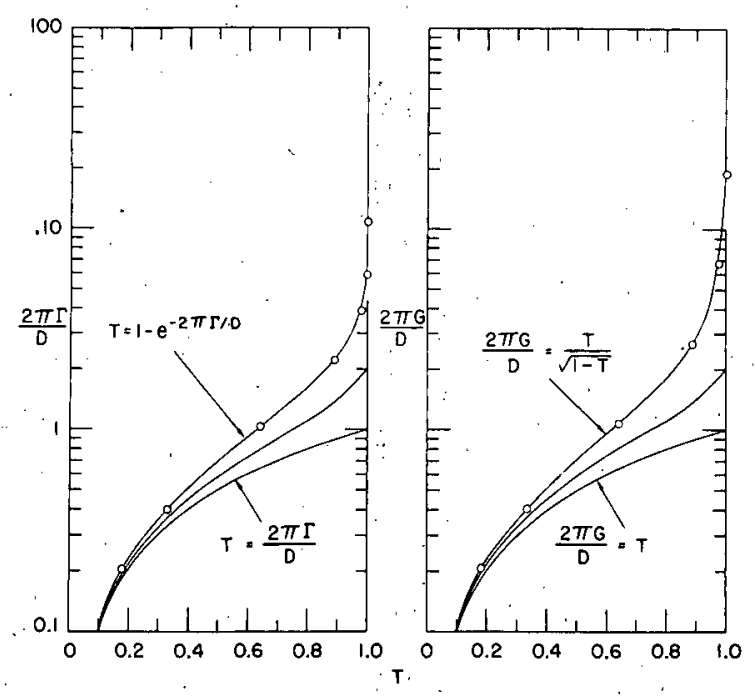

Figure 8 
Figure 9 shows this same set of curves, but now they are compared with the results of the numerical Monte Carlo calculations. These calculations were done for models with 1, 20, and 100 statistically independent channels, all having equal transmission coefficients. In most cases five statistically independent calculations containing 50 poles each were made. These results agree well with the theory. On the right we have plotted the average absolute values of the residues which lie above the curve by an amount which is determined by the dispersion of the phases of the residues. This dispersion of phases is seen to increase with increasing transmission coefficients and also with increasing numbers of open channels.. The numerical results shown become unreliable for $\mathrm{T}>0.9$ because of certain small sample effects.

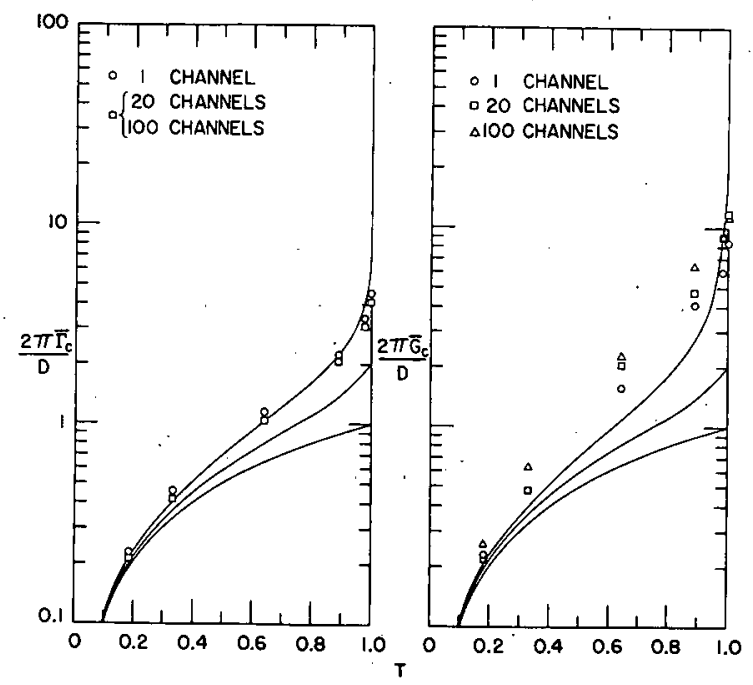

Figure 9

We have now confirmed the results on average resonance pole parameters. But, to calculate average cross sections, and particularly cross section fluctuations, we must also know the distributions of these pole parameters about their averages. These have only been studied with the numerical Monte Carlo calculations so far.

The histograms in Fig. 10 show the distributions of S-matrix pole spacings obtained with various numbers of competing channels, each having $\mathrm{T}=0.89$. The bottom histogram shows the distributions of the K-matrix 
pole spacings which were chosen so as to follow the solid line Wigner distribution. The other histograms show the distributions of resulting S-matrix pole spacings for various numbers of competing channe1s. The repulsion effect is significantly reduced in all the S-matrix histograms and disappears entirely for the cases with large channel numbers where one has completely uncorrelated pole positions as indicated by the dashed exponential spacing distribution law.

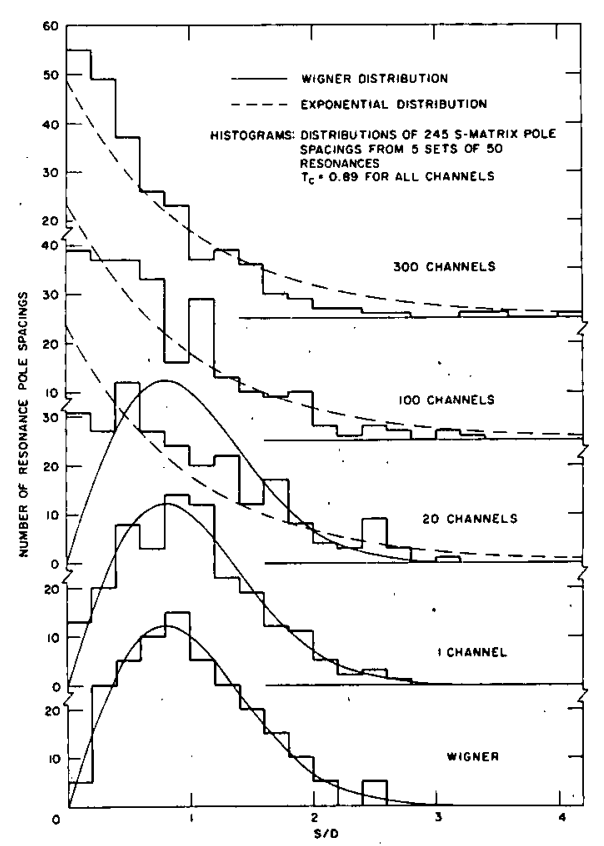

Figure 10

Now we turn to the distribution of widths. The top histogram in Fig. 11 shows the distribution of K-matrix residues which were chosen to follow the Porter-Thomas distribution indicated by the curve. The bottom histogram was obtained from the distribution of S-matrix width in the single channel $\mathrm{T}=0.89$ case. This bottom histogram is broader than the Porter-Thomas distribution, and the difference is statistically significant. This fact becomes more obvious when we look at the many-channel S-matrix width distribution histograms shown in Fig. 12. These results are compared with curves indicating the $x^{2}$. distributions that would be expected if the partial widths had independent Porter-Thomas distributions. 
We see that the actual S-matrix width distributions are much broader. That is, they predict a much larger range of values. The distribution of channel residues is even broader than the distribution of partial widths.

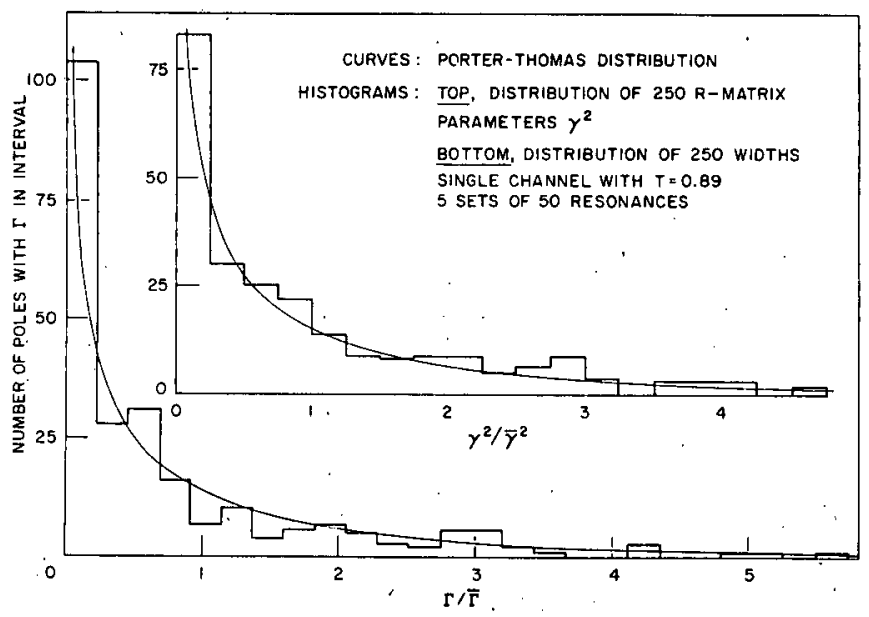

Figure 11

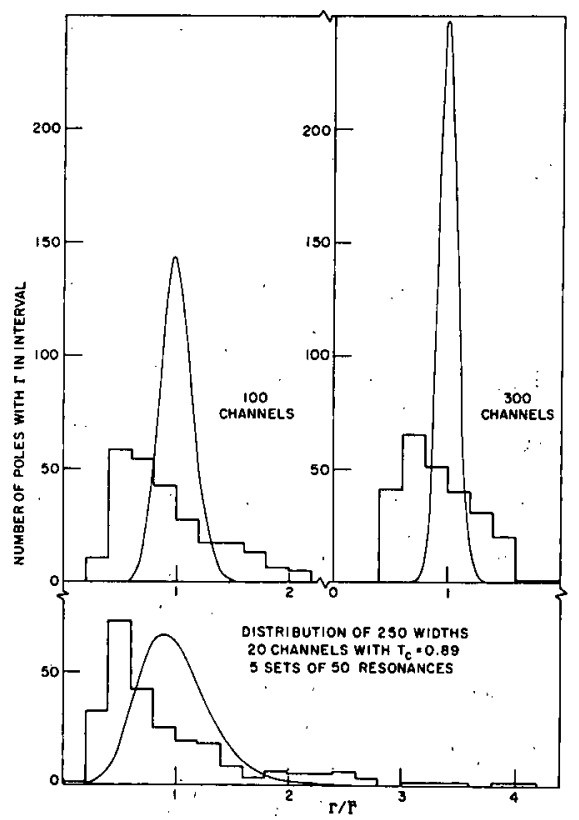

Figure 12 
One consequence of this is that we expect to find an occasional S-matrix pole which has a very large width and also a very large residue in one or more channels. Such a feature will give rise to a broad bump in the average cross section.

\section{CROSS SECTIONS}

This last-mentioned feature is illustrated in Fig. 13 where we show the same Monte Carlo calculations as before, but now the cross section has been averaged over energy interval indicated by the triangle in the top right corner. This average cross section reveals an intermediate structure bump due to that one big pole. Our statistical calculations enable us to predict the frequency of occurrence and other properties of such intermediate structure resonances.

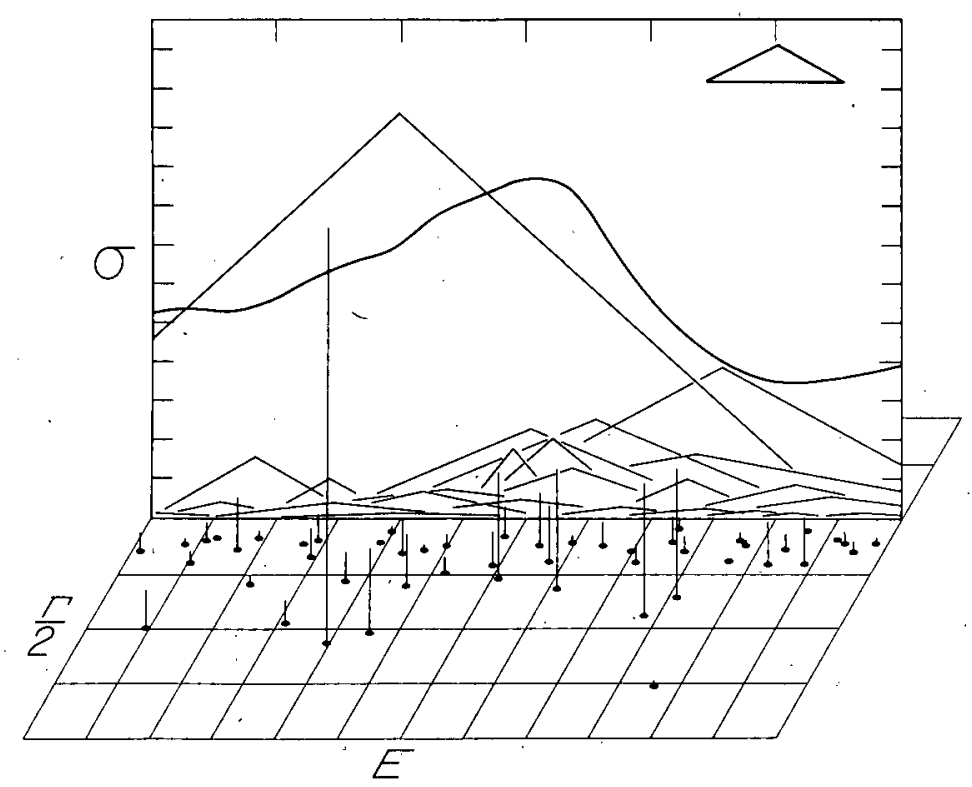

Figure 13

Finally, I want to show the results of several single-channe1 Monte Carlo calculations in which the parameters, that is, the transmission coefficients, were chosen to fit those of nuclei with measured cross sections. Figure 14 shows on the top left the iron scattering cross section measured by Smith at Argonne. The curves on top right are the 
cross section (fine line) and $50 \mathrm{keV}$ averaged cross section (heavy line) obtained by the Monte Carlo method with a transmission coefficient that is appropriate to $1 \mathrm{MeV}$ neutrons. This calculation is not designed to reproduce the long-range energy trend of the cross section. It does, however, agree with the magnitude and fluctuations of the cross section around $1 \mathrm{MeV}$. The bottom curve is the result of the same calculation, but here the S-function pole parameters have been chosen directly according to the usual compound nucleus statistical models instead of being derived from the K-matrix. Both the average cross section and the nature of the fluctuations differ substantially from the experimental results.

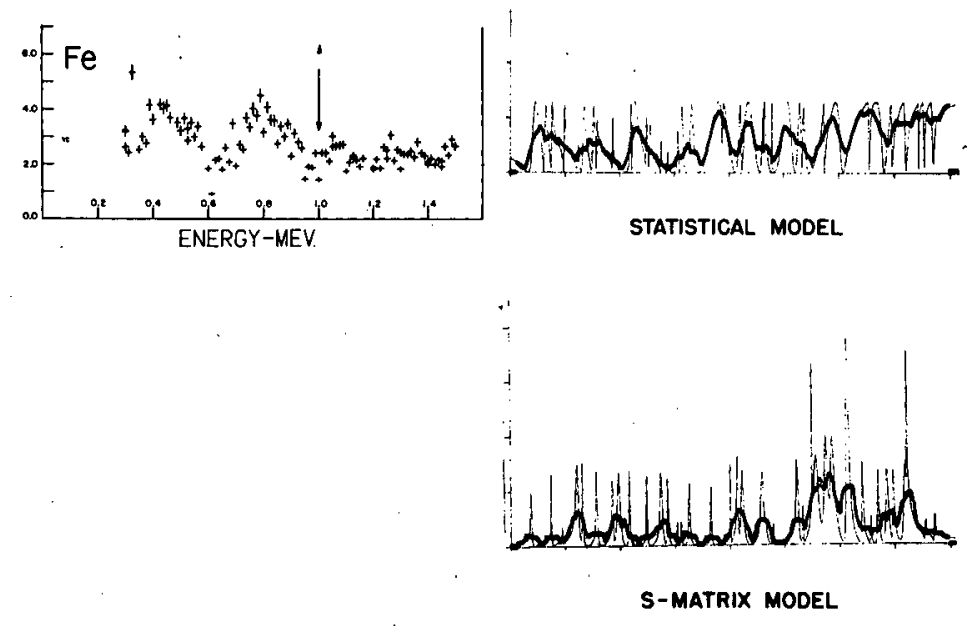

Figure 14

Figure 15 shows statistical model calculations for neutron scattering from manganese and zirconium at $1 \mathrm{MeV}$. The experimental curves on the left were obtained by Cox at Argonne. Two 50-pole statistical sample calculations are shown for each element. The heavy curves, which are the calculations averaged over $50 \mathrm{keV}$, again agree with the magnitudes and the qualitative fluctuations of the data. Furthermore, there are significant differences between the "widths" and "amplitudes" of the cross section fluctuations in these three elements. 


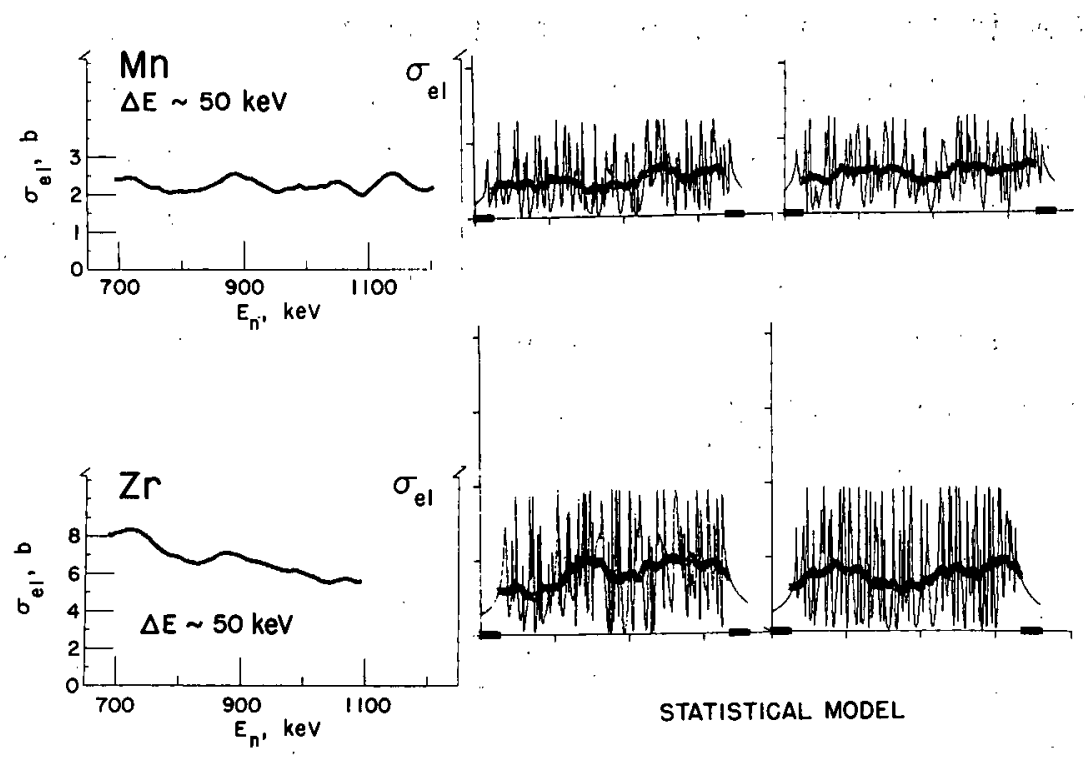

Figure 15

I would conclude that a great deal has been learned about the properties of S-matrix resonance poles. What is now needed is to apply this information to the interpretation and prediction of cross section. This work is now underway.

\section{AVERAGE CROSS SECTIONS}

There has been a good deal of discussion regarding the correct formula for average cross sections. I therefore want to emphasize that none of the parameters which we have discussed so far are directly applicable to average cross section calculations. The average nonelastic cross section for a process going from channel 1 to channel 2 is

$$
\bar{\sigma}_{12}=\pi \pi_{1}{ }^{2}\left\langle\frac{\left|g_{1}\right|^{2} \cdot\left|g_{2}\right|^{2}}{\Gamma}\right\rangle .
$$

It is possible to rewrite this expression in the form

$$
\bar{\sigma}_{12}=\pi_{1}{ }^{2}\left\langle\frac{{ }_{1}^{\theta_{1}}{ }^{\theta_{2}}}{\sum_{i} \theta_{i}}\right\rangle,
$$


where the sum in the denominator is over all channels. The results of the Monte Carlo calculations just described can also be used to determine the statistical properties of $\theta$. Preliminary results indicate that $\langle\theta\rangle$ deviates from $\mathrm{T}$ by no more than $20 \%$ depending on the value of $\mathrm{T}$ and competing channels. The apparent range of values of $\langle\theta\rangle$ is indicated by the shaded region in Fig. 16. More precise results on this and on the fluctuations of $\theta$ will be reported soon.

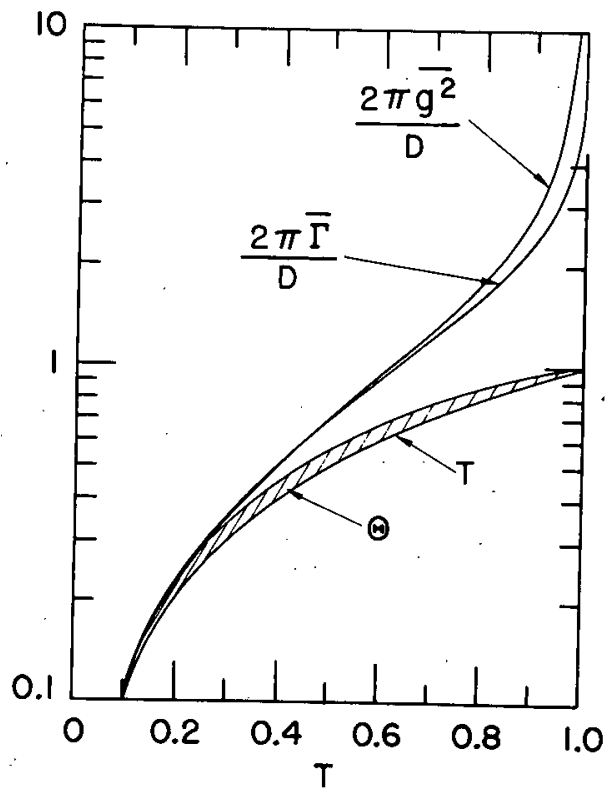

Figure 16

REFERENCES

This paper is based on the following publications where further details and references may be found:

Phys. Rev. 135, B642 (1964).

Rev. Mod. Phys. 36, 1079 (1964).

Phys. Rev. Letters 18, 249 (1967).

Phys. Rev. 157, 907 (1967).

Phys. Rev. Letters 19, 1047 (1967).

Phys. Rev. 171, 1164 (1968). 
Form AEC- 426

Rev. $6 / 68$

AECM 3201
U. S. ATOMIC ENERGY COMMISSION

RECOMRENDATION FOR'DISPOSITION OF INFORMATION PRODUCT

(Washington-designated contractors use Form AEC-427)

\section{- See Instructions on Reverse Side.-}

\section{INTERFERING RESONANCES}

\section{Type of Information Product:}

Xa. Scientific and technical report.

b. Conference paper (if this box is checked, items 5, 6, and 7 below do not apply.) Title of conference

Date of conference

Exact location

Sponsoring organization

$\square$ c. Other (Specify)

\section{Copies Transmitted: (Check one or more applicable boxes)}

Q a. Copies being transmitted for standard distribution by AEC - DTI.

$\square$ b. Copies being transmitted for special distribution by AEC - DTI; per attached list.

$\square$ c. A single reproducible copy being transmitted to AEC - DTI.

\section{Recommended Distribution: (Check one)}

a. Normal handling: no restraint on distribution except as may be required by the security classification and patent clearance status.

Do. Normal handling in the U.S., but there should be no foreign distribution.

Dc. Make available only to U.S. Government agencies and their contractors.

d. Make available only within AEC and to AEC contractors.

$\square$ e. Make available only within AEC.

Df. Make available only to those listed in item 10., below.

Gg. Make available only as specifically approved by the responsible program division.
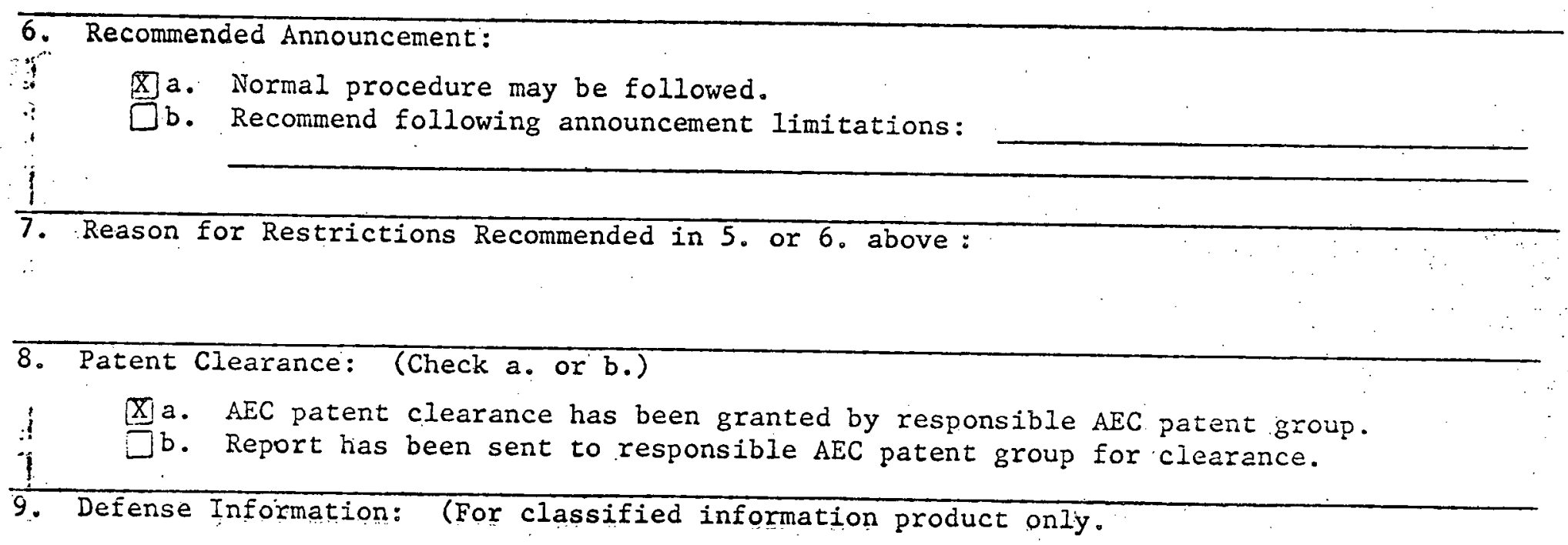

$$
\text { Da. does }
$$

Report

W. dees not

contain defense information other than restricted data.

10. Additional information or remarks. -

11. Submitied by:

Alvin Glassner

Name and position (Please print or type)

Chief Editor

Organilacion Technical Publications Department

Argonne National Laboratory

Sigraicure

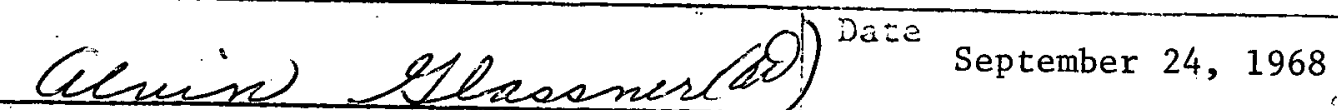

